\title{
Le rôle de fiduciaire des administrateurs de sociétés par actions et la responsabilité sociale des entreprises : la Cour suprême du Canada se prononce dans l'affaire Peoples
}

\author{
Marc-André Morency et Jeanne Simard \\ Université du Québec à Chicoutimi
}

1 e 29 octobre 2004, la Cour suprême du Canada rendait un important jugement concernant la responsabilité fiduciaire des administrateurs de sociétés par actions incorporées en vertu de la Loi canadienne sur les sociétés par actions (LCSA). Le plus haut tribunal du pays mettait ainsi fin à une longue controverse et du même coup posait un cran d'arrêt à une interprétation du texte législatif beaucoup trop restrictive qui avait justement pour effet de faire croire que les administrateurs devaient se soumettre à une règle impérative de poursuite du profit maximum ou de la valeur maximum pour les actionnaires. Cette interprétation très mercantile, exacerbée par la nature même des marchés financiers et la cupidité ambiante, semblait faire de la LCSA un obstacle majeur aux réformes corporatives dans l'esprit de la responsabilité sociale des entreprises. La Cour, dans l'affaire Magasins à rayons Peoples Inc. (syndic de) c. Wise (ciaprès désignée l'affaire Peoples), est intervenue de
La Cour a clarifié la nature des obligations de fiduciaire des administrateurs et dirigeants de sociétés canadiennes, c'est-à-dire leur devoir de loyauté et de diligence en faveur de la personne morale. tion ou la surveillance de la gestion de l'entreprise, pour autant toutefois que cela soit fait au mieux des intérêts de la société par actions. Par contre, la Cour ne va pas jusqu'à adhérer directement au principe de responsabilité sociale qui imposerait aux administrateurs un devoir de loyauté à l'égard des autres parties prenantes. On peut donc estimer que la LCSA peut tenir la route entre deux extrêmes : un mercantilisme débridé à la Friedman, favorable aux seuls actionnaires, et un " socialisme » de la RSE rendant impraticable tout calcul économique rationnel dans l'entreprise.

Pour apprécier pleinement la portée de ce jugement, il convient, avant de présenter les faits à l'origine de cette décision, de faire un bref historique de ce conflit dans l'interprétation des lois corporatives. On pourra ainsi mieux comprendre le difficile arbitrage qui subsiste dans les paramètres décisionnels des sociétés par actions ainsi que le rôle de l'État dans la définition de la responsabilité sociale des entrecontre de cette interprétation et, par conséquent, a clarifié la nature des obligations de fiduciaire des administrateurs et dirigeants de sociétés canadiennes, c'est-à-dire, dans le jargon juridique spécialisé, leur devoir de loyauté et de diligence en faveur de la personne morale.

La Cour a tenu à resituer la place des actionnaires au sein du complexe plus vaste qui compose l'horizon décisionnel des administrateurs en montrant que le devoir de loyauté des administrateurs et des dirigeants n'exclut pas la considération des intérêts des autres parties prenantes, c'est-à-dire les employés, les créanciers, les fournisseurs, les communautés dans la ges- prises. On constatera alors que la décision de la Cour suprême du Canada ne saurait tout régler de cette question et que la vie démocratique conserve toute sa pertinence dans l'orientation de l'économie d'un pays et de ses régions.

\section{La société par actions comme personne morale et l'obligation fiduciaire}

Un bref rappel s'impose. En droit, la « corporation » reçoit, depuis environ 150 ans, le statut d'une personne morale et, à ce titre, elle dispose de capacités juridiques. C'est à cet égard que ses administrateurs et dirigeants se sont vu attribuer des responsabilités. 
L'article 102 de la LCSA précise le pouvoir de gouvernance des administrateurs dans l'entreprise :

Sous réserve de toute convention unanime des actionnaires, les administrateurs gèrent les affaires commerciales et les affaires internes de la société ou en surveillent la gestion.

Cependant, l'article 122 (1) impose des limites à ce pouvoir par une obligation fiduciaire ou de loyauté (au paragraphe a) et une obligation de diligence ( $a u$ paragraphe b) :

Les administrateurs et les dirigeants doivent, dans l'exercice de leurs fonctions, agir :

a) avec intégrité et de bonne foi au mieux des intérêts de la société;

b) avec le soin, la diligence et la compétence dont ferait preuve, en pareilles circonstances, une personne prudente ${ }^{2}$.

L'obligation fiduciaire des administrateurs leur interdit de se mettre en situation de conflit d'intérêts, c'est-à-dire de prendre des décisions à leur propre avantage au détriment de la société par actions. Ils doivent éviter de commettre des abus de pouvoir, de profiter indûment de leur situation d'administrateurs; ils doivent préserver la confidentialité des renseignements auxquels ils ont accès. Selon la Cour d'appel du Québec :

Le devoir de loyauté ou fiduciary duty ne se rapporte pas à la qualité de la gestion des administrateurs, mais plutôt à leur comportement personnel. La loi leur impose d'être loyaux envers ceux qui leur ont confié la mission de gérer les actifs mis en commun. Ce genre de devoir s'intéresse davantage à la motivation des administrateurs plutôt qu'aux conséquences de leurs gestes. Autrement dit, l'intégrité et la bonne foi s'analysent en fonction des raisons qui poussent les administrateurs à agir et non à la lumière des résultats concrets de leurs actions.

L'obligation de diligence, quant à elle, touche le soin attentif, la conduite raisonnable de celui qui prend les décisions. Concrètement, l'obligation de diligence se manifeste par la présence aux réunions du conseil d'administration, l'obligation de se renseigner suffi- samment avant d'agir, de faire enquête s'il y a soupçon d'une fraude d'un administrateur.

\section{L'obligation fiduciaire des administrateurs leur interdit de se mettre en situation de conflit d'intérêts, c'est-à-dire de prendre des décisions à leur propre avantage au détriment de la société par actions.}

\section{Les conflits d'interprétation et les critiques}

Ainsi, lorsqu'ils prennent une décision, les administrateurs doivent, en vertu de leur devoir de fiduciaire, considérer "l'intérêt de la société par actions ". Mais quel est cet intérêt ? Pendant longtemps, la primauté accordée à l'intérêt des actionnaires a exercé une influence marquante sur l'interprétation du devoir de loyauté des administrateurs et des dirigeants, en regard plus particulièrement de l'article 122 de la LCSA. Aussi surprenant que cela puisse paraître, l'obligation qu'impose cet article «d'agir dans l'intérêt de la société par actions ou de la personne morale » a été interprétée majoritairement par la jurisprudence et les auteurs comme signifiant « que les administrateurs et les dirigeants doivent agir dans l'intérêt des actionnaires, véritables bénéficiaires des activités de la société par actions $»^{3}$. Selon un courant jurisprudentiel en common law, une telle interprétation interdirait même aux administrateurs de consacrer des ressources de l'entreprise à des activités charitables. Cette primauté accordée à l'intérêt des actionnaires était largement inspirée par la conception économique néoclassique de l'entreprise qui la définit strictement comme une unité de production (utilisant des intrants pour produire des extrants) pour réaliser, ce faisant, un maximum de profit. Cette vision fait abstraction des aspects légaux, sociaux, politiques de cette forme d'organisation.

La théorie du réseau des contrats particuliers nous aide à saisir un autre aspect de l'organisation, soit la mise en rapport d'acteurs participant à titres divers au processus productif. C'est du moins ce qu'on peut inférer du passage suivant :

[...] il y a un contrat entre les actionnaires et la société lorsqu'il y a souscription ou détention d'actions. Ce contrat oblige les parties à agir de bonne 
foi. Le droit aux dividendes, le droit d'élire les administrateurs, le droit de participer aux assemblées, de se prononcer sur les changements importants de structure sont tous donnés par la loi. Mais l'élément fondamental demeure le contrat de souscription ou d'acquisition d'actions qui est à l'origine de ces droits ${ }^{4}$.

\section{La théorie du réseau des contrats particuliers nous aide à saisir un autre aspect de l'organisation.}

Ces façons restrictives et très mercantiles de concevoir l'entreprise et d'interpréter par ricochet le devoir de fiduciaire des administrateurs ont été critiquées à de nombreuses reprises et à des degrés divers par ceux qui croient qu'une évolution des lois régissant l'obligation fiduciaire est indispensable pour contrer les abus que provoque la règle de la maximisation des profits dans l'intérêt des actionnaires.

En 2002, la Commission sur la démocratie canadienne et la responsabilisation des entreprises, coprésidée par Edward Broadbent et Avie Bennett, déposait un rapport proposant une série de mesures visant à mieux responsabiliser les entreprises, notamment en reconnaissant le principe d'un level playing field et l'élévation du socle légal minimal en matière de protection des parties prenantes. Ces normes minimales, devoirs communs, devraient être respectées aussi bien à l'étranger qu'au plan national, par exemple en matière de respect des normes du travail et de l'environnement $^{5}$. La Commission proposait, entre autres, des amendements importants à la LCSA de façon à préciser la nature exacte des obligations fiduciaires afin que les administrateurs et les dirigeants d'une compagnie soient désormais clairement autorisés à tenir compte de l'impact des activités de l'entreprise sur des groupes d'intérêts non détenteurs d'actions, soit ses employés, ses créanciers, ses clients, ses fournisseurs ainsi que les collectivités locales, en bref ceux qu'on désigne comme des stakeholders, des parties prenantes. Selon la Commission, la loi telle que rédigée et interprétée érige des obstacles artificiels qui empêchent les gestionnaires d'implanter une initiative de RSE rentable à long terme pour la société par actions. La Commission faisait donc la recommandation suivante :
Le droit corporatif devrait être amendé de façon à préciser la nature exacte des obligations fiduciaires des administrateurs à la lumière des principes de RSE. Plus spécifiquement, les lois en question devraient refléter les principes énoncés dans la décision Teck Corporation c. Millar. Il s'agirait ainsi de clarifier le principe suivant. Tant que les administrateurs ne négligent pas entièrement les intérêts des actionnaires au profit d'un intervenant qui ne détient aucune action, ils peuvent tenir compte des enjeux suivants dans le cadre de l'exercice de leurs fonctions et de ce qu'ils jugent le meilleur intérêt de la corporation : a) l'impact des activités de l'entreprise sur ses employés, ses clients, ses fournisseurs et ses créanciers; b) leur impact sur les collectivités où l'entreprise fait affaires; c) les intérêts à court et à long termes de $l^{\prime}$ 'entreprise et des actionnaires ${ }^{6}$.

D'autres critiques vont plus loin et proposent d'élargir la notion de responsabilité fiduciaire aux groupes d'intérêts et de leur accorder un droit de regard sur les décisions de la direction. Ainsi, auraient accès au conseil d'administration des représentants des parties prenantes : syndicats, créanciers, communautés. D'aucuns font remarquer que des créanciers, les banques notamment, ont de fait accès aux conseils d'administration, ce que les syndiqués ne semblent pas rechercher. En droit américain, certains États ont adopté une constituency legislation reconnaissant expressément les intérêts de stakeholders autres que les actionnaires $^{7}$.

Plus récemment, Joël Bacan, professeur de droit à l'Université de la Colombie-Britannique et ancien adjoint du juge Brian Dickson à la Cour suprême du Canada, dénonce, dans son essai intitulé La corporation et dans un film primé portant le même nom, la mission des grandes corporations américaines et canadiennes qui tend à correspondre en bonne partie au modèle idéal d'institution capitaliste défini par Milton Friedman en termes de recherche du profit maximum pour les actionnaires ${ }^{8}$. Selon lui, il faut remodeler la corporation en soumettant son mandat à des dimensions plus spécifiques de la vie sociale et ainsi l'obliger à rendre des comptes aux autres acteurs sociaux. Mais une telle transformation ne pourrait se faire que dans le cadre d'un projet plus vaste de réfection de l'ensemble de l'économie où les gouvernements démocratiques sont invités à assumer leurs responsabilités dans l'encadrement du monde corporatif $f^{\text {' }}$. 
Le but, à long terme, est d'instaurer un ordre économique plus démocratique et plus humain; à court terme, le défi est de trouver des moyens pour contrôler efficacement les entreprises, c'est-à-dire pour les assujettir aux contraintes démocratiques et protéger les citoyens contre leurs tendances. La stratégie la plus appropriée, ou en tout cas la plus réaliste, est de renouveler la légitimité, l'imputabilité et l'efficacité de la réglementation gouvernementale ${ }^{10}$.

D'autres auteurs prétendent que la société par actions a une existence autonome, donc un intérêt distinct qui ne doit même pas prendre en considération les attentes des actionnaires ni des autres parties prenantes de la société! Enfin, de nombreux manifestants, présents sur les lieux des derniers sommets économiques internationaux, exigent une remise en cause draconienne de l'existence ou de la légitimité même des compagnies.

Ces conflits d'interprétation et de critiques faisaient en sorte que le jugement de la Cour suprême du Canada dans l'affaire Peoples était très attendu par les spécialistes et les administrateurs de personnes morales du pays, d'autant plus que le plus haut tribunal du pays ne s'était pas prononcé sur les devoirs des administrateurs depuis plus de 30 ans $^{11}$. Voyons maintenant les faits de l'affaire à l'origine de ce jugement.

\section{L'affaire Peoples}

C'est dans un contexte d'extrême concurrence au sein du commerce de détail que Wise Stores Inc. de Montréal réalisait enfin en 1992 un vieux rêve, celui d'acquérir de Marks \& Spencer Canada Inc., une firme d'origine britannique, les 81 magasins à rayons Peoples. Leur propre chaîne de 50 magasins se trouvait à rejoindre un large réseau de succursales implantées à travers tout le Québec, et de l'Ontario à Terre-Neuve. Les ventes annuelles de Peoples se chiffraient à quelque 160 millions de dollars, mais le réseau connaissait des pertes d'environ 10 millions de dollars annuellement et éprouvait de grandes difficultés financières. L'achat de 180 magasins de Woolco par WalMart n'allait pas peser légèrement dans la suite des choses. On se rappellera les faillites de Continental, Kresge, etc. qui allaient survenir au cours de cette décennie d'implantation des grandes surfaces.
Les frères Wise utilisent alors une formule populaire, celle des achats par effet de levier (leverage buy out ou LBO), c'est-à-dire par endettement, pour procéder à l'acquisition. La convention d'achat d'actions entre les Wise et Marks \& Spencer Canada Inc. porte sur 27 millions de dollars, somme qui doit être versée sur une période de huit ans. Les conditions suivantes sont fixées : Marks \& Spencer Canada Inc. prend les actifs de Peoples en garantie; aucune fusion entre Peoples et Wise ne peut se faire avant la fin du plein paiement à Marks \& Spencer Canada Inc.; Peoples ne doit fournir aucune aide financière à Wise; les opérations financières et l'exploitation doivent demeurer séparées. Dès le début, ces conditions draconiennes mettent la nouvelle chaîne en difficulté. Leurs opérations ne peuvent effectivement fusionner. On doit faire des comptabilités parallèles, tenir séparée la gestion des stocks de marchandise. Wise entreprend néanmoins de rationaliser l'administration. Ces gains de synergie posent des problèmes graves. Les opérations administratives doublent de volume pour certains employés : la gestion de l'entrepôt commun à Ville Saint-Laurent devient un cauchemar en matière d'inventaire.

Les administrateurs de Wise se font alors conseiller par le vice-président à l'administration et aux finances de mettre en œuvre une politique d'approvisionnement commune qui fragmente la responsabilité des achats, Peoples s'occupant des achats en Amérique du Nord et Wise de ceux faits outre-mer. Peoples devait transférer à Wise la marchandise achetée et lui en réclamer le prix, et vice et versa. Comme $82 \%$ de la totalité de la marchandise de Wise et de Peoples est achetée auprès de fournisseurs nord-américains, il en résulte que Peoples consent en apparence un important crédit commercial à Wise soit, au 30 avril 1994, un peu plus de 18 millions de dollars. Pour sa part, le personnel de Wise supporte un fardeau administratif croissant en raison des recherches de synergie et de rationalisation. Cette contrepartie semble avoir été mal évaluée par plusieurs acteurs du procès de première instance, d'où le procès intenté par le syndic et le jugement qui s'ensuivit. L'entreposage et la manutention de la marchandise au dépôt central de Ville Saint-Laurent n'ont pas permis de régler concrètement les problèmes de la gestion matérielle des stocks ni de clarifier les opérations comptables afférentes.

En raison de la grande compétitivité dans le secteur de la vente au détail, et entre autres de l'arrivée sur le marché d'un compétiteur de taille, Wal-Mart, Wise et 
Peoples sont acculés à la faillite à la fin de 1994 devant les exigences de Marks \& Spencer Canada Inc. et le retrait du soutien de crédit par la Banque Toronto Dominion (TD). Les actifs suffisent à rembourser presque entièrement le reliquat de la dette envers Marks \& Spencer Canada Inc. Par contre, d'importantes créances de fournisseurs de Peoples demeurent impayées. Le syndic prétend alors que la politique d'approvisionnement adoptée par les administrateurs de Wise a favorisé les intérêts de Wise plutôt que ceux de Peoples et ce, en contravention des obligations prévues au paragraphe (1) de l'article 122 de la LCSA, soit leurs obligations de fiduciaire et de diligence. La question était donc de savoir si les administrateurs ont une obligation de fiduciaire et une obligation de diligence envers les créanciers de la société. En première instance, le juge Greenberg de la Cour supérieure du Québec ${ }^{12}$ estime que les administrateurs de Wise, sans qu'il y ait eu fraude ou abus, ont manqué de diligence et de loyauté dans la gestion de Peoples, tout particulièrement en adoptant le système d'approvisionnement commun. Les frères Wise se trouvent alors condamnés à verser 6 millions de dollars au syndic de la faillite de Peoples sur la base combinée des articles 100(2) de la Loi sur la faillite et 122 de la LCSA. Le jugement qui a d'importantes conséquences financières pour les Wise et Chubb, leur assureur, est porté en appel. La Cour d'appel du Québec $^{13}$ casse le jugement Greenberg et exprime sa réticence à assimiler les intérêts des créanciers aux intérêts supérieurs de la société. Le syndic porte l'affaire devant la Cour suprême du Canada qui accepte d'entendre la cause et de préciser la nature de l'obligation fiduciaire.

\section{Le rejet du principe de primauté des actionnaires}

Selon la Cour suprême du Canada, la preuve ne démontre pas qu'il y a eu déloyauté de la part des administrateurs de Wise. La Cour semble avoir retenu le concept d'entreprise naissante comme assise de son jugement. Autrement dit, les Wise cherchent à créer une meilleure entreprise. Les « défauts » du processus décisionnel sont donc reliés à la transition en cours, $\mathrm{du}$ fait aussi que les Wise sont, comme administrateurs, dirigeants et actionnaires principaux, les premiers intéressés à réussir dans l'implantation d'un réseau élargi. Le fait qu'il n'y ait pas eu décision formelle au conseil d'administration, aussi bien chez
Peoples que chez Wise relativement au nouveau régime d'achats, n'est pas retenu comme défaut de loyauté. La Cour statue que la firme n'est pas alors nécessairement vouée à la faillite. Les administrateurs de Wise n'avaient pas à agir d'abord en fonction des créanciers, dans la conjoncture donnée. Leur jugement d'affaire n'a pas été remis en question par la Cour. Leur devoir de fiduciaire devait s'exprimer à l'endroit de la personne morale en émergence.

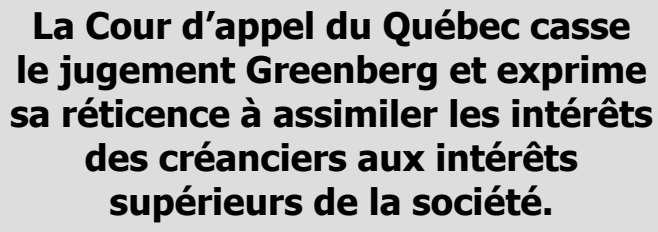

Pour en arriver à cette conclusion, la Cour tient compte des principes énoncés dans une décision d'avantgarde rendue en 1973 par la Cour suprême de Colombie-Britannique (l'équivalent de la Cour supérieure au Québec). Dans cette affaire, on reconnaissait que d'autres facteurs que le simple intérêt des actionnaires pouvait servir à déterminer les éléments que les administrateurs devaient prendre en compte dans une gestion au mieux des intérêts à long terme de la société. En effet, le juge Berger dans l'affaire Teck c. Millar (1972), 33 D.L.R. (3d) 288 (C.S.C.-B.) mentionnait ce qui suit :

Une théorie classique auparavant incontestable doit être abandonnée devant l'évidence des faits de la vie moderne. C'est effectivement ce qui s'est passé. Si, aujourd'hui, les administrateurs d'une société devaient tenir compte des intérêts de ses employés, nul n'alléguerait que, ce faisant, ils n'ont pas agi de bonne foi dans l'intérêt de la société elle-même. De même, si les administrateurs devaient tenir compte des répercussions pour la collectivité d'une politique que l'entreprise a l'intention d'appliquer et ne pouvaient, en conséquence, s'en tenir à cette politique, on ne saurait dire qu'ils n'ont pas tenu compte de bonne foi des intérêts des actionnaires (par. 42).

La Cour suprême du Canada va dans le même sens :

Pour ce qui est de l'obligation fiduciaire prévue par la loi, il est évident qu'il ne faut pas interpréter l'expression « au mieux des intérêts de la société » 
comme si elle signifiait simplement « au mieux des intérêts des actionnaires ». D'un point de vue économique, l'expression « au mieux des intérêts de la société »s'entend de la maximisation de la valeur de l'entreprise (par. 42).

Les juges estiment que les administrateurs manqueraient à leur devoir de fiduciaire s'ils écartaient entièrement les intérêts des actionnaires afin de conférer, par exemple, un avantage à leurs employés. Cependant, en raison des termes mêmes de la LCSA :

Nous considérons qu'il est juste d'affirmer en droit que, pour déterminer s'il agit au mieux des intérêts de la société, il peut être légitime pour le conseil d'administration, vu l'ensemble des circonstances dans un cas donné, de tenir compte notamment des intérêts des actionnaires, des employés, des fournisseurs, des créanciers, des consommateurs, des gouvernements et de l'environnement (par. 42).

Notons que cette position rejoint dans les faits celle que défendait la Commission sur la démocratie canadienne et la responsabilisation des entreprises deux ans plus tôt, mais sans qu'on doive changer d'un iota la loi existante. Ainsi, la Cour retient que les intérêts de la société par actions ne se confondent pas avec ceux des actionnaires, des créanciers ni avec ceux de toute autre partie intéressée. En d'autres termes, contrairement à l'opinion du juge de première instance dans cette affaire, les administrateurs d'une société par actions ne sont pas les fiduciaires des intérêts particuliers des créanciers ou des autres groupes d'intérêts de la société, même pour une entreprise «au bord de la faillite », notion impossible à définir en droit. Cette mise en garde fait ressortir que l'intérêt de la société par actions comme personne morale a été défini par la LCSA comme la valeur suprême par rapport aux autres entités qui composent son environnement. Son existence autonome (et son développement en tant que " meilleure entreprise ») représente le critère prééminent pour ses dirigeants, même si, par ses opérations, elle se trouve affecter l'existence de communautés, de travailleurs, d'employés, de créanciers.

En utilisant leurs compétences au profit de la société lorsqu'elle a des difficultés financières, les administrateurs doivent essayer d'agir au mieux des intérêts de la société en créant une «meilleu- re » société et éviter de favoriser les intérêts d'un groupe d'intéressés en particulier (par. 47).

Par conséquent, on peut s'interroger si, dans les faits, cette décision de la Cour, et l'interprétation qu'elle fait de l'article 122(1)(a) de la LCSA, sensibiliseront davantage les gestionnaires à la responsabilité sociale des entreprises, à l'éthique des affaires, au respect des déontologies professionnelles, au respect des lois en général, au Canada et à l'étranger. Cette interprétation plus précise de l'obligation fiduciaire aura certainement le mérite de faire disparaître l'épée de Damoclès qui semblait s'opposer à une éventuelle initiative de prise en compte des intérêts des autres parties prenantes de la société par actions, en atténuant la crainte de poursuites éventuelles. D'autres mesures pourraient par contre être nécessaires pour qu'une nouvelle culture d'entreprise prenne la place qui lui revient dans le monde contemporain. Les questions éthiques et sociales sont souvent perçues comme des freins au mandat premier de la compagnie qui est de maximiser la valeur de l'entreprise. Pour ce qui est des autres parties prenantes, si leurs droits sont brimés, quels sont les recours disponibles dans la LCSA et le C.c.Q. ?

\section{Les juges estiment que les administrateurs manqueraient à leur devoir de fiduciaire s'ils écartaient entièrement les intérêts des actionnaires afin de conférer, par exemple, un avantage à leurs employés.}

\section{Les recours}

Comme la Cour suprême du Canada l'a bien démontré, la société par actions est la seule destinataire du devoir de loyauté des administrateurs. Par conséquent, elle seule peut intenter un recours direct s'il y a contravention à ce devoir; les autres parties prenantes n'ayant pas de recours direct contre les administrateurs en cas de violation de ces devoirs. Cependant, la Cour a fortement rappelé la possibilité pour deux parties prenantes, les actionnaires et les créanciers touchés par les opérations corporatives, de se faire reconnaître comme plaignants, par recours indirect, le recours pour oppression ou abus de droit, aussi prévu dans la LCSA. En effet, l'article 241(2)(c) autorise un tribunal à accorder un redressement si : 
[...] la société ou l'une des personnes morales de son groupe [...] abuse des droits de détenteurs de valeurs mobilières, créanciers, administrateurs ou dirigeants, ou se montre injuste à leur égard en leur portant préjudice ou en ne tenant pas compte de leurs intérêts :

[...] soit par la façon dont ses administrateurs exercent ou ont exercé leurs pouvoirs ${ }^{14}$.

Ainsi, les actionnaires et les créanciers pourront, en cas de comportements abusifs et injustes à leur égard de la part de la société par actions ou de ses administrateurs, obtenir la protection de leurs intérêts en recourant au tribunal qui a, en pareil cas, des pouvoirs quasi illimités de redressement. Ce recours n'est pas ouvert aux autres parties prenantes. "C C'est donc dire qu'un employé congédié par la société ne bénéficie pas à ce titre du recours, même s'il est par ailleurs actionnaire ou administrateur $\gg{ }^{15}$.

Les autres parties prenantes devront donc se rabattre sur un recours en responsabilité extracontractuelle figurant aux articles 1457 du Code civil du Québec et 122(1)(b) de la LCSA. Lus ensemble, ces articles prévoient que les administrateurs et les dirigeants doivent, dans l'exercice de leurs fonctions, « agir... avec le soin, la diligence et la compétence dont ferait preuve, en pareilles circonstances, une personne prudente » et ce, de manière à ne pas causer de préjudice à autrui. Pour savoir s'il y a eu une faute de diligence de la part des administrateurs, les tribunaux examinent s'ils ont pris une décision raisonnable et non pas la meilleure décision. Selon la Cour suprême du Canada :

[...] dès lors que la décision prise conserve un caractère raisonnable, le tribunal ne devrait pas substituer son avis à celui du conseil [d'administration], même si les événements ultérieurs peuvent avoir jeté le doute sur la décision du conseil (par. 65).

La Cour se réfère explicitement à la réserve des tribunaux vis à vis des jugements d'affaires (business judgment rule), estimant que les administrateurs sont généralement, dans des circonstances données, et avec des données imparfaites, capables de décider au mieux des intérêts corporatifs. Par conséquent, ce recours est considérablement restreint, car il devient très difficile pour une partie prenante d'établir que les

administrateurs ont manqué à leur obligation de diligence.

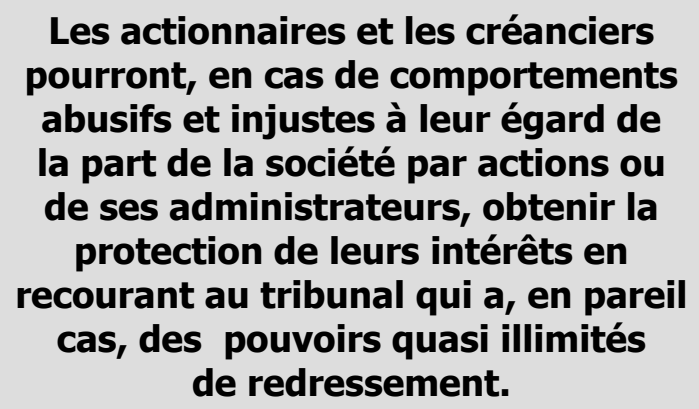

\section{Conclusion}

Le cadre décisionnel des administrateurs de sociétés par actions canadiennes, tel qu'interprété par la Cour suprême du Canada, a naturellement une portée très large puisqu'elles opèrent aux États-Unis et dans le monde entier. Seul l'État peut, s'il n'abdique pas sa responsabilité propre, indiquer aux sociétés par actions, ainsi qu'à l'ensemble des acteurs sociaux, le socle commun des observances et règles propres à assurer le bien-être commun sans lequel la vie sociale ne peut exister. L'État peut le faire, pour la collectivité, s'il conserve évidemment son pouvoir de contrainte et de sanction. Ce que la mondialisation néolibérale portée par les grandes corporations cherche à éroder.

L'application du principe du level playing field, c'està-dire l'expression de devoirs communs aux entreprises édictés par l'État et la démocratie, est d'une importance capitale mais se trouverait menacée s'il y avait abdication et " course à la hausse » au recrutement corporatif, comme c'est le cas au Delaware ${ }^{16}$. L'intention du Québec, annoncée par le ministre des Finances, de déréglementer l'incorporation mérite d'être suivie de très près, sinon la concurrence à la baisse au sein de l'ordre juridique canadien pourrait sacrifier la RSE sur l'autel corporatif et aurait pour effet d'annuler les avancées canadiennes en matière de droit des affaires.

\section{Remerciements}

Les auteurs tiennent à remercier le professeur Stéphane Rousseau de l'Université de Montréal pour ses judicieux commentaires et monsieur Roger de la Sablonnière pour l'aide apportée en matière documentaire. Au moment de mettre sous presse, nous prenons connaissance de la publication de la Revue 
canadienne du droit du commerce intitulée «Symposium on the Supreme Court's Judgment in the Peoples Department Stores Case ». Voir le vol. 41, p. 167 à 246.

\section{Notes biographiques}

Marc-André Morency est professeur de sociologie au Département des sciences humaines à l'Université du Québec à Chicoutimi.

Jeanne Simard est avocate et professeure de droit au Département des sciences économiques et administratives à l'Université du Québec à Chicoutimi.

\section{Notes et références}

1 Magasins à rayons Peoples inc. (syndic de) c. Wise, Cour suprême du Canada, 29 octobre 2004.

2 Au provincial, les différentes lois qui régissent les compagnies traitent de ces obligations avec un libellé semblable. Entre autres, les articles 321 et 322 du Code civil du Québec spécifient ceci : «L'administrateur est considéré comme mandataire de la personne morale. Il doit, dans l'exercice de ses fonctions, respecter les obligations que la loi, l'acte constitutif et les règlements lui imposent et agir dans les limites des pouvoirs qui lui sont conférés ». « L'administrateur doit agir avec prudence et diligence. Il doit aussi agir avec honnêteté et loyauté dans l'intérêt de la personne morale. » L'article 123.83 de la Loi québécoise sur les compagnies, quant à lui, mentionne : «Les administrateurs, officiers et autres représentants de la compagnie sont considérés comme des mandataires de la compagnie ».

3 Crête, R. et S. Rousseau (2003). Droit des sociétés par actions; principes fondamentaux, Montréal, Éditions Thémis, p. 83.

4 Morisset, A. et J. Turgeon (2004). Droit corporatif canadien et québécois, Farnham, Publications CCH Ltée, 2 vol. (f. mobiles), p. 21-110.

5 Morency, M.-A. et J. Simard (2003). « La responsabilité sociale des entreprises : vers un nouveau consensus canadien ", revue Organisations et territoires, vol. 12, $\mathrm{n}^{\circ} 2$, p. 101-108.

6 Commission sur la démocratie canadienne et la responsabilisation des entreprises, Rapport final : une nouvelle équation; les profits et les responsabilités des entreprises à l'aube du $21^{e}$ siècle, janvier 2002, p. 24. Ce document peut être consulté à l'adresse suivante :

http://www.corporate-accountability.ca.

7 Rousseau, S. (1997). «Chronique bibliographique », $\mathrm{Ca}$ hier de droit, vol. 38, p. 462-467; Martel, P. (2001), « Les devoirs de loyauté des administrateurs de sociétés par actions fédérales - impact du Code civil du Québec », Revue du Barreau, tome 61, p. 323-394.

8 Bacan (2004), op. cit.

9 Pour une analyse plus poussée de cette perspective dans le cadre européen, voir; Aglietta, M. et A. Rebérioux (2004). Dérives du capitalisme financier, collection présentée par R.M. Solow, Paris, Éditions Albin Michel, 394 p.; Lordon, F. (2003). Et la vertu sauvera le monde... Après la débâcle financière, le salut par l'«éthique»?, Paris, Éditions Raisons d'agir, $125 \mathrm{p}$.

10 Bacan (2004), op. cit., p. 175.

11 Gray, W.D. (2003), « Peoples v. Wise and Dylex : Identifying Stakeholder Interests Upon or Near Corporate Insolvency - Stasis or Pragmatism ? », Canadian Business Law Journal, vol. 39, p. 242-261.

12 [1999] R.R.A. 178 (C.S.) (résumé).

13 [2003] R.J.Q. 796 à 818 (C.A.).

14 La personne qui demande un redressement en cas d'abus de droit doit, de l'avis de la Cour suprême du Canada, être visée par la définition de "plaignant» que l'on trouve à l'article 238 de la LCSA : a) le détenteur inscrit ou le véritable propriétaire, ancien ou actuel, de valeurs mobilières d'une société ou de personnes morales du même groupe; b) tout administrateur ou dirigeant, ancien ou actuel, d'une société ou de personnes morales du même groupe; c) le directeur; d) toute autre personne qui, d'après un tribunal, a qualité pour présenter les demandes visées à la présente partie.

15 Martel, M. et P. Martel (2005). Les compagnies au Québec. Les aspects juridiques, Montréal, Les Éditions Wilson \& Lafleur, Martel, Ltée, p. 31-83.

16 Poulin-Goyer, G. (2005). « Droit commercial : une chirurgie plastique aux traits du Delaware. La souplesse de l'État américain intéresse le Québec », Le Devoir, 10 janvier 2005 , p. B-1. 